\title{
Passivity-based Output Synchronization of Dynamical Networks with Non-identical Nodes
}

\author{
Jun Zhao, David J.Hill and Tao Liu
}

\begin{abstract}
Output synchronization of dynamical networks with non-identical nodes is studied using the passivity property. A synchronization criterion is developed for networks with general outer coupling topologies which need to be neither symmetric nor have the zero-row-sum property. When the passivity property cannot give synchronization for a single outer coupling topology, the problem of how to achieve synchronization by switching among several outer coupling topologies is studied. Synchronization conditions by switching among these topologies are presented and an output-dependent switching law is designed. In particular, it is shown that synchronizability can be checked by verifying if a certain nonlinear programming problem has no feasible solution or has a negative maximum.
\end{abstract}

\section{INTRODUCTION}

Synchronization problems of dynamical networks occur in a variety of contexts, having extensive applications in physics, biology, ecology and many engineering fields [1], [3], [15], [23], [29]. Focus of the study has been mainly put on networks with identical nodes. Indeed, identical node dynamics greatly simplify the synchronization analysis. In this aspect, the master stability function approach plays a dominating role in the study of local synchronization. In particular, identical node dynamics together with a constant, symmetric and irreducible coupling configuration matrix always makes it possible to locally transform the network dynamics into a set of decoupled lower-dimensional systems whose stability can be easily determined by analyzing the master stability function [1], [3]. Many extensions of this technique have been also available in the literature [2], [7], [16], [25], [31] - see also recent survey papers [1], [3] and references therein.

Global synchronization of dynamical networks is of course a very preferable property but is a very difficult issue and few results have been reported until now. Some results are still based on the stability of the master stability equation (see for instance, [12], [13], [33]). In these results, the nonlinear dynamics of each node are linearized and the stability margin given by the master stability equation

This work was supported by the Australian Research Council's Discovery Projects and Centres of Excellence Schemes (Projects FF 0455875 and CEO 348217), and NNSF of China under Grants 60874024 and 90816028, and the SRFDP of China under Grant 200801450019.

J.Zhao is with Key Laboratory of Integrated Automation of Process Industry, Ministry of Education of China, Northeastern University, Shenyang, 110819, PR China, and is also with School of Engineering, The Australian National University, Canberra ACT 0200, Australia.

D.J.Hill is with School of Engineering, The Australian National University and National ICT Australia, Canberra ACT 0200, Australia.

T.Liu is with School of Engineering, The Australian National University, Canberra ACT 0200, Australia. jun. zhaodanu.edu.au, David.Hilleanu.edu.au, tao.liu@anu.edu.au method for the linearized network is assumed to be large enough to compensate the nonlinear terms which are treated as perturbations satisfying linear growth conditions.

Often, some strong properties of the node dynamics have to be imposed in order to have global synchronization without linearization. The notion of $V$-uniformly decreasing functions was proposed in [29] and a full dimensional synchronization criterion was set up. Another property that is required to guarantee global synchronization is the $V$ stability assumption of each node [30]. A similar method was applied in [24].

The assumption of networks having identical nodes is not always realistic. In fact, most dynamical networks in engineering have different nodes. Examples can be found in many practical networks, such as power systems [9] and multi-robot systems [14]. The study of synchronization of dynamical networks with non-identical nodes is very hard and very few results have been reported by now. Several collective properties for coupled non-identical chaotic systems were respectively discussed in [8], [26], [28]. A simulation study for non-identical Kuramoto oscillators was carried out in [4]. Controlled synchronization was considered for the case that each node has a normal form with a linear main part [18], and distributed controllers were designed to achieve synchronization. When asymptotic synchronization is impossible for networks with non-identical nodes, partial or bounded synchronization were studied instead [22], [32]. Looking for effective methods for networks with nonidentical nodes is a challenging issue.

On the other hand, passivity is a useful system property that has been widely exploited in analysis and synthesis of nonlinear control systems [27]. Since a dynamical network is composed of a number of nodes that are nonlinear systems, it is natural and reasonable to expect passivity to be useful for network analysis and design. In fact, several attempts using the passivity property to deal with synchronization problems have been made. An output synchronization condition for multi-agent systems was proposed when the outer coupling is given by a constant multiplied by differences between node outputs [5]. A similar method was used for networks with time delay in communication [6]. A weaker version of passivity, called semi-passivity, was shown to be able to create ultimate boundedness of the solutions [21]. For a certain class of coupled chaotic systems, passivity can produce a synchronization feature [11]. There are some relevant works including flocking algorithm in $\mathrm{SE}(3)$ [10], global analysis of limit cycles of interconnected oscillators [20] and applications to the analysis of biochemical networks 
[19].

In this paper, we study the synchronization problem for dynamical networks with non-identical nodes using passivity. With respect to previous work on the topic, the main contributions of this paper are as follows. First of all, a general output synchronization criterion based on passivity is developed with no symmetry or zero-row-sum property of the outer coupling matrix assumed. Therefore, the proposed criterion is well-suited to a more general class of networks. Secondly, with help of passivity, output synchronization is achieved by switching among a number of outer coupling topologies, with each of them, if used alone, synchronization may not be achieved. This strategy has not been seen in the literature so far. Thirdly, a nonlinear programming problem is formulated to check synchronizability of networks with switching topologies.

\section{Preliminaries}

Consider a network composed of $N$ non-identical nodes. Each isolated node is represented by the nonlinear control system

$$
\begin{aligned}
& \dot{x}_{i}=f_{i}\left(x_{i}\right)+g_{i}\left(x_{i}\right) u_{i}, \\
& y_{i}=h_{i}\left(x_{i}\right), \quad i=1,2, \cdots, N,
\end{aligned}
$$

where $x_{i}=\left(x_{i 1}, x_{i 2}, \cdots, x_{i n}\right)^{T} \in R^{n}, u_{i}, y_{i} \in R^{m}$ are the state variable, control input and output of the $i$-th node, respectively; $f_{i}: R^{n} \rightarrow R^{n}, g_{i}: R^{n} \rightarrow R^{n \times m}$ and $h_{i}:$ $R^{n} \rightarrow R^{m}$ are continuous mappings.

$$
\begin{aligned}
& \text { Choose } x=\left(\begin{array}{c}
x_{1} \\
\vdots \\
x_{N}
\end{array}\right), f(x)=\left(\begin{array}{c}
f_{1}\left(x_{1}\right) \\
\vdots \\
f_{N}\left(x_{N}\right)
\end{array}\right), g(x)= \\
& \left(\begin{array}{cccc}
g_{1}\left(x_{1}\right) & 0 & \cdots & 0 \\
0 & g_{2}\left(x_{2}\right) & \cdots & 0 \\
\cdots & \cdots & \cdots & \cdots \\
0 & 0 & \cdots & g_{N}\left(x_{N}\right)
\end{array}\right), u=\left(\begin{array}{c}
u_{1} \\
\vdots \\
u_{N}
\end{array}\right), \\
& y=\left(\begin{array}{c}
y_{1} \\
\vdots \\
y_{N}
\end{array}\right)=h(x)=\left(\begin{array}{c}
h_{1}\left(x_{1}\right) \\
\vdots \\
h_{N}\left(x_{N}\right)
\end{array}\right) .
\end{aligned}
$$

Putting all the independent nodes together makes a nonlinear control system in a compact form

$$
\begin{aligned}
& \dot{x}=f(x)+g(x) u, \\
& y=h(x) .
\end{aligned}
$$

The network is then formed by choosing control action which interconnects nodes through an outer coupling topology

$$
u_{i}=\sum_{j=1}^{N} a_{i j} \Gamma y_{j},
$$

or equivalently $u=(A \otimes \Gamma) y$, where $A=\left(a_{i j}\right)_{N \times N}$ is a matrix, called outer coupling matrix, and $\Gamma$ is an $m \times m$ positive definite matrix, called the inner coupling matrix and $\otimes$ stands for the Kronecker product.
A typical form of the control is given by

$$
u_{i}=\sum_{j=1, j \neq i}^{N} a_{i j} \Gamma\left(y_{j}-y_{i}\right)
$$

which can be rewritten as the form of (3) by defining $a_{i i}=$ $-\sum_{j=1, j \neq i}^{N} a_{i j}$. By doing so, the outer coupling matrix $A$ has the zero row sum property, which is often used as an ideal property. Here, we adopt the general form of $A$ where the zero row sum property need not hold.

Under the interconnection (3), the network can be expressed by

$$
\begin{aligned}
& \dot{x}_{i}=f_{i}\left(x_{i}\right)+g_{i}\left(x_{i}\right) \sum_{j=1}^{N} a_{i j} \Gamma y_{j}, \\
& y_{i}=h_{i}\left(x_{i}\right), \quad i=1,2, \cdots, N,
\end{aligned}
$$

or equivalently,

$$
\begin{aligned}
\dot{x} & =f(x)+g(x)(A \otimes \Gamma) y . \\
y & =h(x) .
\end{aligned}
$$

The network (4) is said to achieve output synchronization if

$$
\lim _{t \rightarrow \infty}\left(y_{i}\left(x_{i}(t)\right)-y_{j}\left(x_{j}(t)\right)\right)=0, i, j=1, \cdots, N
$$

Obviously, output synchronization is equivalent to the convergence of $x(t)$ to the output synchronization manifold:

$$
M=\left\{x \mid h_{1}\left(x_{1}\right)=h_{2}\left(x_{2}\right)=\cdots=h_{N}\left(x_{N}\right)\right\} .
$$

Since we are interested in the problem of how to have output synchronization by the passivity property, we make the following assumption.

Assumption 2.1. As a dynamic control system, each node of (1) is passive.

From this assumption and according to the KYP lemma [27], there exist storage functions $V_{i}\left(x_{i}\right)$, which are positive definite, such that

$$
\begin{gathered}
L_{f_{i}} V_{i}\left(x_{i}\right) \leq 0, \\
L_{g_{i}} V_{i}\left(x_{i}\right)=h_{i}^{T}\left(x_{i}\right) .
\end{gathered}
$$

\section{SYNCHRONIZATION CRITERION}

In this section, we will establish a general output synchronization criterion using the passivity property.

Theorem 3.1 (i) If $A+A^{T}$ is negative definite, then $x(t)$ converges to the set

$$
\operatorname{ker}(h)=\left\{x \mid h_{i}\left(x_{i}\right)=0, i=1,2, \cdots, N\right\},
$$

which implies output synchronization;

(ii) If $A+A^{T}$ is semi-negative definite with a simple zero eigenvalue, then, there exist constants $\alpha_{i}, i=1,2, \cdots, N$ with $\sum_{i=1}^{N} \alpha_{i}^{2}=1$ such that $x(t)$ converges to the set

$$
\left\{x \mid \alpha_{i} h_{j}\left(x_{j}\right)-\alpha_{j} h_{i}\left(x_{i}\right)=0, i, j=1,2, \cdots, N\right\} ;
$$

(iii) If $A+A^{T}$ has the zero row sum property and has $N-$ 1 negative eigenvalues, then, $x(t)$ converges to the output synchronization manifold $M$. 
Proof. Choose $V(x)=\sum_{i=1}^{N} V_{i}\left(x_{i}\right)$. Differentiating $V$ along the trajectory of the network (4) and using passivity we have

$$
\begin{aligned}
2 \dot{V} & =2 \sum_{i=1}^{N} L_{f_{i}} V_{i}+2 \sum_{i=1}^{N} L_{g_{i}} V_{i} u_{i} \\
& \leq 2 \sum_{i=1}^{N} h_{i}^{T} u_{i} \\
& =2 y^{T}(A \otimes \Gamma) y \\
& =y^{T}\left(\left(A+A^{T}\right) \otimes \Gamma\right) y .
\end{aligned}
$$

(i). Since $A+A^{T}$ is negative definite and $\Gamma$ is positive definite, $\left(A+A^{T}\right) \otimes \Gamma$ is negative definite. Therefore, $\dot{V}<0$ if $h(x) \neq 0$ and $V=0$ if and only if $h(x)=0$. By Lasalle's Invariance Principle, $x(t)$ converges to the set $\operatorname{ker}(h)$.

(ii). Choose a unitary matrix $\Phi=\left(\Phi_{1}, \cdots, \Phi_{N}\right)$ with its columns $\Phi_{i}=\left(\phi_{1 i}, \phi_{2 i} \cdots, \phi_{N i}\right)^{T}$ such that

$$
\Phi^{T}\left(A+A^{T}\right) \Phi=\operatorname{diag}\left\{\lambda_{1}, \cdots, \lambda_{N}\right\}
$$

where $\lambda_{i}$ are eigenvalues of $A+A^{T}$ satisfying

$$
0=\lambda_{1}>\lambda_{2} \geq \lambda_{3} \geq \cdots \geq \lambda_{N} .
$$

Let $z=\left(\Phi^{T} \otimes I_{m}\right) y$. Then, it follows from (9) that

$$
\begin{aligned}
2 \dot{V} & \leq z^{T}\left(\Phi^{T} \otimes I_{m}\right)\left(\left(A+A^{T}\right) \otimes \Gamma\right)\left(\Phi \otimes I_{m}\right) z \\
& =z^{T} \operatorname{diag}\left\{\lambda_{1} \Gamma, \cdots, \lambda_{N} \Gamma\right\} z \\
& =\sum_{i=2}^{N} \lambda_{i} z_{i}^{T} \Gamma z_{i} .
\end{aligned}
$$

Thus, $\dot{V}<0$ if $\left(z_{2}^{T}, \cdots, z_{N}^{T}\right)^{T} \neq 0$, and $\dot{V}=0$ if and only if $\left(z_{2}^{T}, \cdots, z_{N}^{T}\right)^{T}=0$, which in turn results in

$$
\left(\begin{array}{cccc}
\phi_{11} I_{m} & * & \cdots & * \\
\phi_{21} I_{m} & * & \cdots & * \\
\cdots & & \cdots & \cdots \\
\phi_{N 1} I_{m} & * & \cdots & *
\end{array}\right)\left(\begin{array}{c}
z_{1} \\
0 \\
\vdots \\
0
\end{array}\right)=\left(\begin{array}{c}
\phi_{11} z_{1} \\
\phi_{21} z_{1} \\
\vdots \\
\phi_{N 1} z_{1}
\end{array}\right)
$$

Let $\alpha_{i}=\phi_{i 1}$. Thus, $\dot{V}=0$ only if

$$
\alpha_{i} h_{j}\left(x_{j}\right)-\alpha_{j} h_{i}\left(x_{i}\right)=0, i, j=1,2, \cdots, N .
$$

Applying Lasalle's Invariance Principle again gives the result.

(iii). The zero row sum property of $A+A^{T}$ allows us to choose $\Phi$ in (ii) with $\Phi_{1}=\left(\frac{1}{\sqrt{N}}, \frac{1}{\sqrt{N}}, \cdots, \frac{1}{\sqrt{N}}\right)^{T}$. Then output synchronization follows from (ii).

Remark 3.2. When the interconnection is given by $u_{i}=$ $\sum_{j \in \mathcal{N}_{i}} k\left(y_{j}-y_{i}\right)$, where $k$ is a positive constant and $\mathcal{N}_{i}$ is the set of nodes transmitting their outputs to the $i$-th node, and if the graph representing the outer coupling topology is balanced, then, output synchronization is achieved. Therefore, the result of [5] is a special case of Theorem 3.1.

Corollary 3.3. If $A+A^{T}$ is negative definite and the network (4), if viewed as a dynamical system, is zero-state detectable, then the network (4) realizes state synchronization.
Proof. Applying zero-state detectability to (9) immediately produces state synchronization.

Corollary 3.4. If $A+A^{T}$ is irreducible, has the zero row sum property and all off-diagonal entries are non-negative, then, the network (4) achieves output synchronization.

Proof. Obvious.

Remark 3.5. We have global synchronization results in the sense of global convergence in Theorem 3.1 if all the storage functions $V_{i}$ are radially unbounded.

\section{Synchronization By Switching}

If the matrix $A+A^{T}$ is neither negative definite nor semi-negative definite with the zero row sum property and with a simple zero eigenvalue, we can not ensure output synchronization from passivity. In this case, it is natural to ask if passivity still plays an important role in checking output synchronization. The answer is positive under certain circumstances if we are given a number of applicable outer coupling topologies such that with each of them output synchronization cannot be guaranteed by passivity. More precisely, if we are allowed to use $l$ outer coupling matrices $A_{k}=\left(a_{i j}^{k}\right)_{N \times N}, k=1,2, \cdots, l$, and to switch among them and each $A_{k}$, if used alone, can not guarantee output synchronization, output synchronization is still possible if we choose the switching among these $A_{k}$ properly. In this section, we will show how to achieve output synchronization by design the switchings. Of course, none of $A_{k}$ is assumed to satisfy (i) or (iii) in Theorem 3.1 because otherwise it will be trivial to choose a constant switching law.

Theorem 4.1. If there exist constants $\beta_{k} \geq 0, k=$ $1, \cdots, l$, such that $A=\sum_{k=1}^{l} \beta_{k} A_{k}$ satisfies either (i) or (iii), then output synchronization can be achieved by switchings.

Proof. If $A$ satisfies (i), the proof is obvious. We now suppose $A$ satisfies (iii). Using a similar argument as in the proof of Theorem 3.1, we can easily show

$$
y^{T}\left(\left(A+A^{T}\right) \otimes \Gamma\right) y=0 \Leftrightarrow x \in M .
$$

Thus, for any $x \notin M$,

$$
\sum_{k=1}^{l} \beta_{k} y^{T}\left(\left(A_{k}+A_{k}^{T}\right) \otimes \Gamma\right) y=y^{T}\left(\left(A+A^{T}\right) \otimes \Gamma\right) y<0,
$$

which implies that for each $y$ with $x \notin M$, there exists at least one $k$ such that $y^{T}\left(\left(A_{k}+A_{k}^{T}\right) \otimes \Gamma\right) y<0$. Design the switching law as

$$
\sigma=\sigma(h(x))=\arg \min \left\{h^{T}(x)\left(\left(A_{k}+A_{k}^{T}\right) \otimes \Gamma\right) h(x)\right\} .
$$

Then,

$$
\dot{V} \leq h^{T}(x)\left(\left(A_{\sigma}+A_{\sigma}^{T}\right) \otimes \Gamma\right) h(x)<0, \forall x \notin M .
$$

Once again, applying Lasalle's Invariance Principle completes the proof.

When such a matrix $A$ does not exist or is difficult to find, we need to establish other output synchronization conditions. To this end, we first extend the notion of completeness of a family of functions [17] to completeness on a set. 
Definition 4.2. Let $S=\left\{s_{1}(x), \cdots, s_{p}(x)\right\}$ be a family of functions defined on a subset $\Omega \subset R^{n}$. S is called complete on $\Omega$ if for any $x \in \Omega$ there exists at least one $k$ satisfying $s_{k}(x) \leq 0$. Moreover, $S$ is called strictly complete on $\Omega$ if $s_{k}(x)<0$.

Remark 4.3. The classic notion of completeness (see [17]) is a special case of the above definition when $\Omega=R^{n} \backslash\{0\}$.

Proposition 4.4. If the family of functions $S=$ $\left\{h^{T}(x)\left(\left(A_{k}+A_{k}^{T}\right) \otimes \Gamma\right) h(x), k=1, \cdots, l\right\}$ is strictly complete on $R^{n} \backslash M$, then output synchronization is achieved by the switching law (16).

Proof. Obvious.

This proposition gives a principle of checking output synchronization. In order to make this principle implementable, we need to develop methods to check the strict completeness

For any fixed $i, 1 \leq i \leq l$, consider the nonlinear programming problem

$$
\begin{array}{cl}
\max & w^{T}\left(\left(\bar{A}_{i}+\bar{A}_{i}^{T}\right) \otimes \Gamma\right) w \\
\text { s.t. } & w^{T}\left(\left(\bar{A}_{j}+\bar{A}_{j}^{T}\right) \otimes \Gamma\right) w \geq 0,1 \leq j \leq l, j \neq i, \\
& w^{T} w=1,
\end{array}
$$

where $w \in R^{m(N-1)}$,

$$
\bar{A}_{i}=\left(\begin{array}{cccc}
a_{22}^{i} & a_{23}^{i} & \cdots & a_{2 N}^{i} \\
a_{32}^{i} & a_{33}^{i} & \cdots & a_{3 N}^{i} \\
\vdots & \vdots & \ddots & \vdots \\
a_{N 2}^{i} & a_{N 3}^{i} & \cdots & a_{N N}^{i}
\end{array}\right)
$$

which is the matrix obtained by deleting the first row and the first column of the matrix $A_{i}$.

The output synchronization problem is closely related to the nonlinear programming problem above, as shown in the following theorem.

Theorem 4.5. Suppose all $A_{k}+A_{k}^{T}$ have the zero row sum property. If for some $i$ the nonlinear programming problem (18) has no feasible solution or the maximum is negative if a feasible solution exists, then, output synchronization is achieved by the switching law (16).

Proof. For any $y \in R^{m N}$, we have

$$
y=\left(\begin{array}{c}
y_{1} \\
y_{2} \\
\vdots \\
y_{N}
\end{array}\right)=\left(\begin{array}{c}
y_{1} \\
y_{1} \\
\vdots \\
y_{1}
\end{array}\right)+\left(\begin{array}{c}
0 \\
w
\end{array}\right)
$$

where $y_{i} \in R^{m}$ and $w=\left(\begin{array}{c}y_{2}-y_{1} \\ y_{3}-y_{1} \\ \vdots \\ y_{N}-y_{1}\end{array}\right) \in R^{m(N-1)}$.

The zero row sum property ensures

$y^{T}\left(\left(A_{k}+A_{k}^{T}\right) \otimes \Gamma\right) y=w^{T}\left(\left(\bar{A}_{k}+\bar{A}_{k}^{T}\right) \otimes \Gamma\right) w, k=1, \cdots, l$.

Obviously, $x \in M$ if and only if $w=0$.

We split the proof into two cases.
Case 1: (18) has no feasible solution. In this case,

$$
\left\{\begin{array}{l}
w^{T}\left(\left(\bar{A}_{j}+\bar{A}_{j}^{T}\right) \otimes \Gamma\right) w \geq 0,1 \leq j \leq l, j \neq i, \\
w^{T} w=1
\end{array}\right.
$$

has no solution, which means by normalizing $w$ that

$$
\left\{\begin{array}{l}
w^{T}\left(\left(\bar{A}_{j}+\bar{A}_{j}^{T}\right) \otimes \Gamma\right) w \geq 0,1 \leq j \leq l, j \neq i, \\
w \neq 0
\end{array}\right.
$$

has no solution. Therefore, for any $w \neq 0$ there exists at least $j$ such that

$$
w^{T}\left(\left(\bar{A}_{j}+\bar{A}_{j}^{T}\right) \otimes \Gamma\right) w<0 .
$$

Applying (19) we know that for any $x \notin M$ there exist $j$ such that

$$
h^{T}(x)\left(\left(A_{j}+A_{j}^{T}\right) \otimes \Gamma\right) h(x)<0,
$$

which indicates that $S=\left\{h^{T}(x)\left(\left(A_{k}+A_{k}^{T}\right) \otimes \Gamma\right) h(x), k=\right.$ $1, \cdots, l\}$ is strictly complete on $R^{n} \backslash M$, and thus output synchronization follows from Proposition 4.4.

Case 2: The maximum of (18) is negative. For any $y=$ $h(x)$ with $x \notin M$, if

$$
y^{T}\left(\left(A_{j}+A_{j}^{T}\right) \otimes \Gamma\right) y \geq 0, j \neq i,
$$

then (19) gives

$$
\left\{\begin{array}{l}
w^{T}\left(\left(\bar{A}_{j}+\bar{A}_{j}^{T}\right) \otimes \Gamma\right) w \geq 0, j \neq i \\
w \neq 0 .
\end{array}\right.
$$

Set $\bar{w}=\frac{w}{\|w\|}$. Then,

$$
\left\{\begin{array}{l}
\bar{w}^{T}\left(\left(\bar{A}_{j}+\bar{A}_{j}^{T}\right) \otimes \Gamma\right) \bar{w} \geq 0, j \neq i, \\
\bar{w}^{T} \bar{w}=1 .
\end{array}\right.
$$

The negative maximum of (18) guarantees

$$
\bar{w}^{T}\left(\left(\bar{A}_{i}+\bar{A}_{i}^{T}\right) \otimes \Gamma\right) \bar{w}<0,
$$

which is equivalent to

$$
w^{T}\left(\left(\bar{A}_{i}+\bar{A}_{i}^{T}\right) \otimes \Gamma\right) w<0 .
$$

According to (19), (28) implies

$$
y^{T}\left(\left(A_{i}+A_{i}^{T}\right) \otimes \Gamma\right) y<0,
$$

which in turn gives rise to the strict completeness of $S=$ $\left\{h^{T}(x)\left(\left(A_{k}+A_{k}^{T}\right) \otimes \Gamma\right) h(x), k=1, \cdots, l\right\}$ on $R^{n} \backslash M$, and thus output synchronization follows from Proposition 4.4.

\section{EXAMPLE}

In this section, we give an example to validate the proposed methods. Consider five coupled pendula as shown in Fig 1.

The dynamics of isolated pendula are

$$
\begin{aligned}
\dot{x}_{i 1} & =x_{i 2}, \\
\dot{x}_{i 2}+\frac{g}{L_{i}} \sin \left(x_{i 1}\right) & =u_{i}, \quad i=1,2,3,4,5, \\
y_{i} & =x_{i 2},
\end{aligned}
$$

which are passive with the storage functions

$$
V_{i}\left(x_{i}\right)=\frac{1}{2} x_{i 2}^{2}+\frac{g}{L_{i}}\left(1-\cos \left(x_{i 1}\right)\right) .
$$




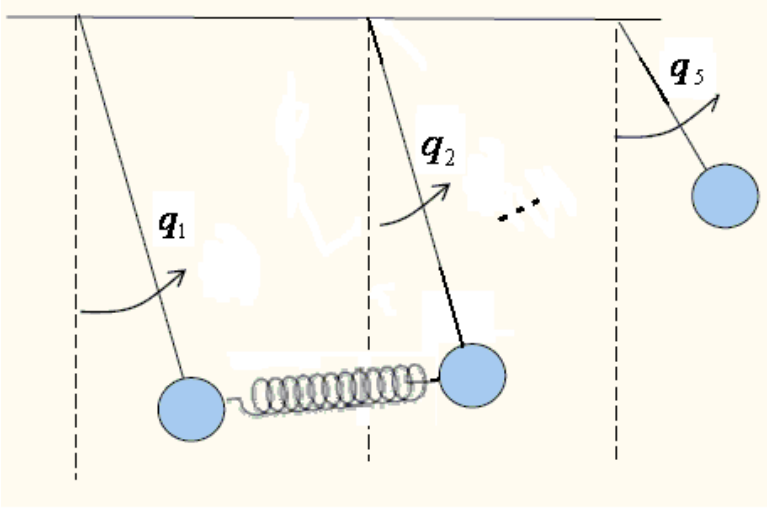

Fig. 1. Coupled pendula.

Let $\Gamma=1, L_{1}=1.05, L_{2}=1.10, L_{3}=1.15, L_{4}=1.20$, $L_{5}=1.25$ and

$$
\begin{aligned}
& A= \\
& \left(\begin{array}{cccccccccc}
-2 & 0 & 1 & -1 & 0 & 0 & 1 & 0 & 0 & 0 \\
0 & -3 & -1 & 1 & 0 & 1 & 0 & 0 & 0 & 1 \\
0 & 0 & -2 & 0 & 1 & 0 & 0 & 0 & 1 & 0 \\
0 & 0 & 1 & -2 & 1 & -1 & 0 & 1 & 0 & 0 \\
0 & -1 & 0 & 0 & -2 & 0 & 0 & 0 & 1 & 0 \\
0 & 0 & 1 & 0 & 0 & -2 & 1 & 0 & 0 & 0 \\
0 & 0 & 0 & 0 & 1 & 0 & -3.5 & 1 & 0 & 1 \\
0 & 0 & 0 & 1 & 0 & 0 & 1 & -2.5 & 1 & 0 \\
0 & 0 & 0 & 1 & 0 & 1 & 0 & 1 & -5 & 1 \\
0 & 0 & 0 & 0 & 0 & 0 & 0 & 0 & 1 & -1.5
\end{array}\right) .
\end{aligned}
$$

It is easy to see that $A+A^{T}<0$. Applying (i) of Theorem 3.1. we know $y_{i} \rightarrow 0$. The simulation result is shown in Fig.2. Now, we consider the same coupled pendula

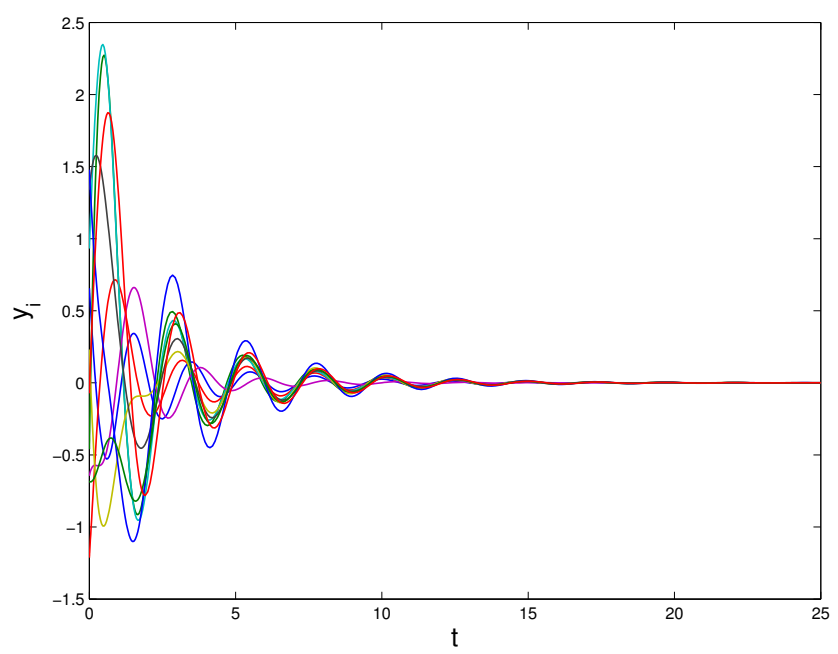

Fig. 2. The outputs $y_{i}$ of non-switching case.

as Example 1 but with three switching topologies given by

$$
A_{1}=\left(\begin{array}{cccccccccc}
0 & 0 & 1 & 0 & -1 & 0 & 0 & 0 & 0 & 0 \\
0 & -4 & 1 & 1 & 0 & 1 & 1 & 0 & 0 & 0 \\
1 & 1 & -2 & 0 & 0 & 0 & 0 & 0 & 0 & 0 \\
0 & 1 & 0 & -1 & 0 & 0 & 0 & 1 & 0 & -1 \\
-1 & 0 & 0 & 0 & 0 & 0 & 0 & 1 & 0 & 0 \\
0 & 1 & 0 & 0 & 0 & -2 & 0 & 1 & 0 & 0 \\
0 & 1 & 0 & 0 & 0 & 0 & -2 & 1 & 0 & 0 \\
0 & 0 & 0 & 1 & 1 & 1 & 1 & -3 & -1 & 0 \\
0 & 0 & 0 & 0 & 0 & 0 & 0 & -1 & 1 & 0 \\
0 & 0 & 0 & -1 & 0 & 0 & 0 & 0 & 0 & 1
\end{array}\right),
$$

$\Gamma=1.5, L_{i}$ are the same as Example 1 .

It is easy to show that if any topology is exploited alone, the network does not output synchronize. A direct computation shows that the nonlinear programming (18) has the negative maximum. By Theorem 4.5, the network achieves output synchronization under the switching law (16), which is depicted in Fig.3-4.

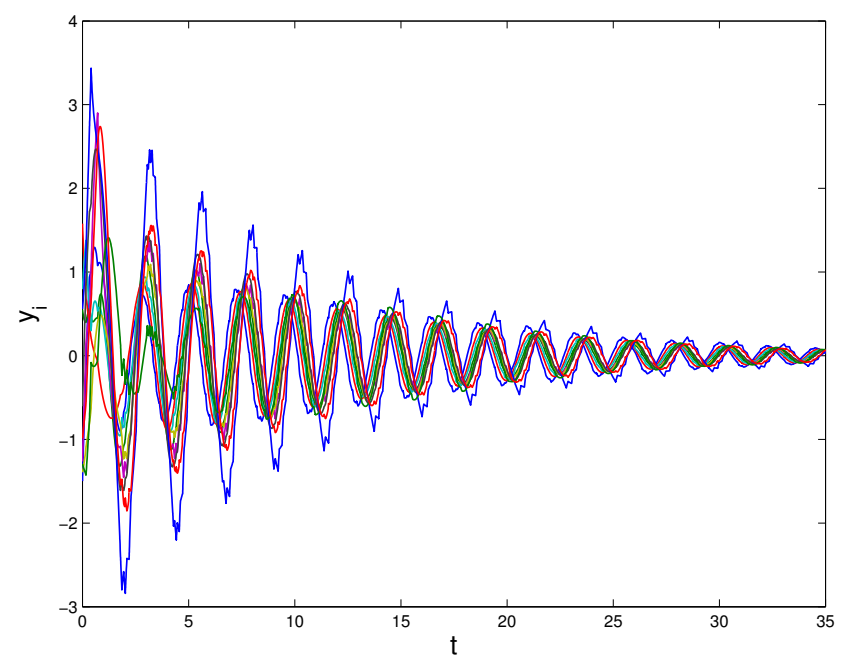

Fig. 3. The outputs $y_{i}$ of the switched network. 


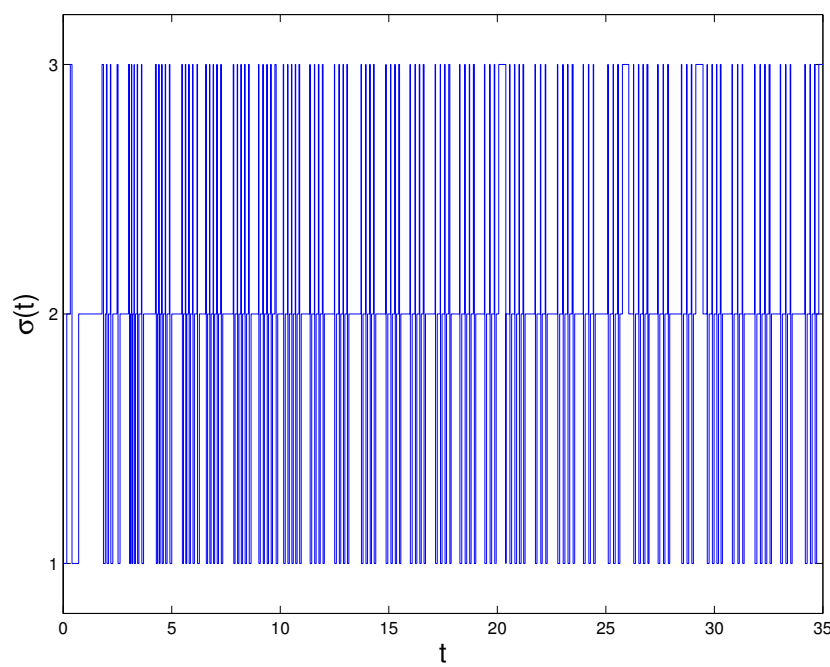

Fig. 4. The switching signal $\sigma(t)$ of the switched network.

\section{CONCLUSIONS}

A output synchronization criterion for dynamical networks with non-identical nodes and with general outer coupling topologies has been presented using the passivity property. A switching strategy is developed for the case of multitopologies when none of the topologies alone may lead to synchronization. The proposed conditions can be checked by computing the sign of the optimal solution of a certain nonlinear programming problem.

The idea of achieving synchronization by designing switching laws based on the passivity property has first appeared in this paper, which enlarges the possibility of synchronizability even if each given outer coupling topology cannot bring synchronization. Turning the synchronization condition into a certain nonlinear programming problem makes it possible to make use of the methods in operational research.

Unlike for networks with identical nodes, we have known little about behaviors of networks with non-identical nodes. Effective approaches need developing to cope with such networks. Passivity has proved useful but is still expected to be able to deal with more problems in the area of dynamical networks.

\section{REFERENCES}

[1] A. Arenas, A. Daz-Guilera, J. Kurths, Y. Moreno and C. Zhou, Synchronization in complex networks, Physics Reports, vol. 469, no. 3, 93-153, 2008.

[2] I.Belykh, V.N.Belykh, M.Hasler, Generalized connection graph method for synchronization in asymmetrical networks, Physica D, Nonlinear Phenomena, vol. 224, no. 1-2, 42-51, 2006.

[3] S.Boccaletti, V.Latora, Y.Moreno, M.Chavez, D.U.Hwang, Complex networks: structure and dynamics, Phys. Rep. 424, 175-308, 2006.

[4] M.Brede, Synchrony-optimized networks of non-identical Kuramoto oscillators, Physics Letters A, vol.372, no.15, 2618-2622, 2008.

[5] N.Chopra, M.W.Spong, Passivity-based control of multi-agent systems, In: Kawamura, S., Svinin, M. (eds.) Advances in Robot Control: From Everyday Physics to Human-Like Movements, Springer, Heidelberg, 107-134, 2006.

[6] N.Chopra, M.W.Spong, Output synchronization of nonlinear systems with time delay in communication, Proc. 45th IEEE conf. Decision and Control, 4986-4992, Sangiego, CA, 2006.
[7] P.DeLellis, M. diBernardo and F. Garofalo, Novel decentralized adaptive strategies for the synchronization of complex networks, Automatica, vol.45, no.5, 1312-1318, 2009.

[8] R.Femat, L.Kocarev, L.van Gerven and M.E.Monsivais-Perez, Towards generalized synchronization of strictly different chaotic systems, Physics Letters A, 342, 247-255, 2005.

[9] D.J.Hill, G.Chen, Power systems as dynamic networks, Proc. IEEE International Symposium on Circuits and Systems, 722-725, 2006.

[10] Y.Igarashi, T.Hatanaka, M.Fujita, M.W.Spong, Passivity-based output synchronization and flocking algorithm in SE(3), Proc. American Control Conference, Seattle, Wangshongton, 723-728, 2008.

[11] K.Kemih, M.Benslama, H.Baudrand, Passivity-based synchronization of unified chaotic system, Journal of Control Science and Engineering, vol.2008, doi:10.1155/2008/567807.

[12] Z.Li, G.Chen, Global synchronization and asymptotic stability of complex dynamical networks, IEEE Trans. Circuits and Systems-II, 53, 28-33, 2006.

[13] J.Lu, D.W.C.Ho, Local and global synchronization in general complex dynamical networks with delay decoupling, Chaos, Solitons \& Fractals vol.37, no.5,1497-1501, 2008.

[14] S.Mastellone, D.Lee, M.W.Spong, Master-slave synchronization with switching communication through passive model-based control design, Proc.American Control Conference, 3203-3208, 2006.

[15] M. Newman, A-L. Barabasi, D.J. Watts, The Structure and Dynamics of Networks, Princeton University Press, Princeton, 2006.

[16] M.Porfiri, D.J.Stilwell, E.M.Bollt, Synchronization in Random Weighted Directed Networks, IEEE Transactions on Circuits and Systems I: Regular Papers,, vol. 55 , no. 10, 3170 - 3177, 2008

[17] A.Pogromsky, M.Jirstrand, P.Spangeus, On Stability and passivity of a class of hybrid systems, Proc. 37th Conf. Decision and Control, Tampa, Florida, 3705-3710, 1998.

[18] J.G.B.Ramirez, R.Femat, On the controlled synchronization of dynamical networks with non-identical nodes, Proc. 3rd International IEEE Scientific Conference on Physics and Control (Physcon 2007), 2007.

[19] L.Scardovi, M.Arcak, E.D.Sontag, Synchronization of interconnected systems aith applications to biochemical networks: an input-output approach, IEEE Trans. Automatic Control, vol.55, 2010 (to appear, available on line).

[20] Guy-Bart Stan, R.Sepulchre, Analysis of interconnected oscillators by dissipativity theory, IEEE Trans. Automa. Contr., vol.52, no.2, 256270, 2007.

[21] E.Steur, I.Tyukin, H.Nijmeijer, Semi-passivity and synchronization of diffusively coupled neuronal oscillators, Physica D, vol.238, 2119$2128,2009$.

[22] J.Sun, E.M.Bolt and T.Nishikawa, , Master Stability Functions for Coupled Near-Identical Dynamical Systems, Euro. Phys. Lett. vol.85, 60011. arXiv:0811.0649v1 [nlin.CD], 2009.

[23] S.H.Strogatz, Exploring complex networks, Nature, vol.410, 268-276, 2001.

[24] Q.Song, J.Cao, F.Liu, Synchronization of complex dynamical networks with nonidentical nodes, Physics Letters A, vol.374, 544-551, 2010.

[25] H.G.Tanner, A.Jadbabaie, G.J.Pappas, Flocking in fixed and switching networks, IEEE Trans. Automatic Control, 52:863-868, 2007.

[26] R.K.Upadhyay, V.Rai, Complex dynamics and synchronization in two non-identical chaotic ecological systems, Chaos, Solitons and Fractals, (available on line).

[27] A.Van der Schaft, $L_{2}$-Gain and Passivity Techniques in Nonlinear Control, Springer, 2000.

[28] U.E.Vincent, J.A.Laoye, Synchronization, anti-synchronization and current transports in non-identical chaotic ratchets, Physica A, 384, 230-240, 2007

[29] C.W.Wu, Synchronization in Complex Networks of Nonlinear Dynamical Systems, World Scientific, Singapore, 2007.

[30] J.Xiang, G.Chen, On the V-stability of complex dynamical networks, Automatica, 43, 1049-1057, 2007.

[31] J.Yao, Z.Guan, D.J.Hill, Passivity-based control and synchronization of general complex dynamical networks, Automatica, vol.45, 2107$2113,2009$.

[32] D.J.Hill and J.Zhao, Global synchronization of complex dynamical networks with non-identical nodes, Proc. CDC, 817-822, 2008.

[33] J.Zhao, D.J.Hill and T.Liu, Synchronization of complex dynamical networks with switching topology: a switched system point of view, Automatica, 2502-2511, 2009. 